\title{
Improved through-wall radar imaging using modified Green's function-based multi-path exploitation method
}

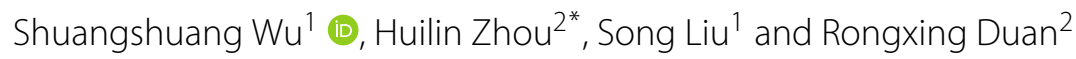

\begin{abstract}
The existence of walls surrounding targets leads to multi-path return at the radar receiver, which provides additional information about the target, and thus can be exploited to strengthen the quality of through-wall radar imaging (TWRI). Based on this, a multi-path exploitation method is proposed to identify the location of the multi-path ghost. An algorithm that combining the modified Green's function with back projection algorithm is presented to associate and map the multi-path ghosts to the location of real targets. The theoretical analysis is verified according to the simulation results obtained using gprMAX software as well as practical radar measured data, and our proposed method is shown to outperform that in conventional multi-path exploitation method (Setlur et al., IEEE Trans. Geosci. Remote Sens. 49:4021-4034, 2011).
\end{abstract}

Keywords: Modified Green's function, Multi-path association and mapping, Through-wall radar imaging

\section{Introduction}

Through-wall radar imaging (TWRI) has attracted much attention in various fields, due to its numerous civil and military application [1-4]. The targets of the throughwall radar are those objects that are located inside rooms surrounded by walls. This leads to the superimposing of multi-path returned signals and the direct returned signal. In the traditional narrowband radar system, it is difficult to separate the original returned signal of target with the those caused by multi-path due to the poor range resolution and the time-delay resolution [5]. Alternatively, ultra-wide band (UWB) through-wall radar systems [6] can provide sufficient range resolution to differentiate the direct and the multi-path returned signal. In the meantime, the multi-path return caused by walls can in turn enlarge the investigation domain and enhance the number of the effective array aperture, if sufficiently separated from the direct return, which motivates the multi-path exploitation method [7].

In $[8,9]$, the algorithm of back propagation (BP) and delay-summing beamforming were proposed to realize

\footnotetext{
${ }^{*}$ Correspondence: zhouhuilin@ncu,edu,cn

${ }^{2}$ School of Information and Engineering, Nanchang University, 330031 Nanchang, People's Republic of China

Full list of author information is available at the end of the article
}

the target refocusing, respectively. However, the interference produced by multi-path cannot be suppressed by the above approaches, which results in the emergence of multi-path ghosts in the image region. In order to solve these problems, several methods were proposed to remove ghosts produced by multi-path [10,11]. An adaptive CLEAN algorithm was proposed to remove the false targets or ghosts based on the BP imaging algorithm [10]. From the perspective of multi-sensor fusion, a distributed algorithm which restrains the ghosts introduced by multipath was proposed in [11] based on the algorithm of fully polarimetric beamforming. The analysis was verified by using data collected from multiple polarimetric orientations. However, the methods proposed in [10-12] regarded multi-path return as clutter, and multi-path was not particularly exploited or analyzed.

Recently, a group sparse compressive sensing approach was used to reconstruct stationary scenes $[13,14]$. The returned signal from the target and walls were separated and shown in the image by using a sparse reconstruction approach, which jointly uses the wall and target models. Moreover, the sparse reconstruction and representation methods are also widely used in various fields of image processing [15-19]. On the basis of the local maximum values extract method and the 1-D Kalman filter, the authors extracted the 1-D trajectories of the real target 
and the multi-path ghosts in each receiving channel in $[20,21]$. Then, based on the principle of the first-order multi-path echoes, the position of the sidewall was computed in each frame, and the multiple frame average value is used to improve the detection accuracy. Besides, instead of using multiple-input multiple-output (MIMO) antenna array, a limited number of transceivers mounted on robot are used [22, 23].

In this paper, the multi-path effect is exploited to reduce false positives and signal-to-clutter ratio (SCR) at the target location by mapping the ghost targets back to the real target position. We propose a calculation model of modified Green's function based on the non-line-of-sight (NLoS) propagation path. After that, Back Projection (BP) imaging combined with modified Green's function is used to obtain the composite image. In order to associate and map the ghosts, an association matrix is constructed, which has deep nulls at the assumed ghosting locations and a large peak value at the real target location, which helps removing the ghosts and ensuring the accuracy of target location. Comparing to the existing literature [7], our simulation results are shown to achieve about $3 \mathrm{~dB}$ SCR gain, which demonstrates the superiority of our proposed method.

The remainder of the paper is organized as follows. The multi-path propagation model is introduced in Section 2. In Section 3, we present a multi-path exploitation algorithm by using the modified Green's function imaging. Section 4 reports representative simulation results of the proposed method, and concluding remarks follow in Section 5.

Through this paper, matrix is denoted by boldface capital letters, $\mathcal{C}$ denote the complex number set, and $\mathbf{A} \in$ $\mathcal{C}^{N \times M}$ denotes a complexed-value matrix of dimension $N \times M$. Moreover, $\mathbf{A}(x, y)$ denotes A's $(x, y)$ th element. The vector is denoted by boldface lowercase letters $x$ and $\boldsymbol{x}=(x, y)$ denotes the position vector of object with $x$ - and $y$-axis coordinates being $x$ and $y$, respectively. A triangle with vertices $A, B$, and $C$ is denoted by $\triangle A B C$ with edges $\mathrm{AB}, \mathrm{BC}$, and $\mathrm{AC}$. The symbol $\perp$ means perpendicularity between two edges. The operator $\lfloor x\rfloor$ denotes the largest integer smaller than $x$.

\section{System model}

In this section, the multi-path system model where we perform through-wall radar imaging is introduced in details. In Section 2.1, we first describe the physical scenario, including the geometric positions of walls and target. Then, under this scenario setup, we obtain the signal model considering the multi-path returns.

\subsection{Scenario description}

A two-dimensional multi-path propagation model is illustrated in Fig. 1, where a target locates inside homogeneous walls, with the relative permittivity, electric conductivity, and thickness of the walls being $\varepsilon_{1}, \sigma_{1}$, and $d_{1}$, respectively. The length of front and the back are the same and denoted by $D_{1}$, while the length of the side walls are $D_{2}$. It is assumed that the transmitter and receiver are ideal dipoles and centered at the same position $\mathrm{R}_{n}$ with position vector $\boldsymbol{r}_{n}=\left(-x_{n}, y_{n}\right), n=1,2, \cdots, N$, where $N$ is the number of antennas along the wall. The target is located at position $\boldsymbol{x}_{\mathrm{t}}=\left(-x_{\mathrm{t}}, y_{\mathrm{t}}\right)$ behind the front wall. It is assumed that the bandwidth is sufficient wide such that direct or multi-path returns can be resolved.

\subsection{Multi-path signal propagation model}

In Fig. 1, both the direct path as well as three indirect paths are considered. The first-order multi-paths are defined as the signal returned through the indirect path together with the path A or vice versa, while the second-order multi-path is defined as the signal that reaches the target and returns back to the transceivers by the indirect way. Let the time-varying transmitted signal denoted by $s(t)$, then the composite received signal at the $n$th sensor by the superposition of the direct path and multi-path returns is given as

$$
\begin{aligned}
r_{n}(t)= & A_{0} s\left(t-2 \tau_{\mathrm{A}}^{(n)}\right)+\sum_{p \in\{\mathrm{B}, \mathrm{C}, \mathrm{D}\}} A_{p, 1} s\left(t-\left(\tau_{\mathrm{A}}^{(n)}+\tau_{p}^{(n)}\right)\right) \\
& +\sum_{p \in\{\mathrm{B}, \mathrm{C}, \mathrm{D}\}} A_{p, 2} s\left(t-2 \tau_{p}^{(n)}\right)
\end{aligned}
$$

where $A_{0}, A_{p, 1}$, and $A_{p, 2}$ are the complex amplitudes related to the direct reflection and transmission coefficients for the direct path, first-order multi-path, and second-order multi-path of the $p$ th path, respectively, where $p \in\{\mathrm{B}, \mathrm{C}, \mathrm{D}\}$. Moreover, $\tau_{\mathrm{A}}^{(n)}$ and $\tau_{p}^{(n)}$ are the singlebounce multi-path delays of the returned signals through path $A$ and paths $B, C$, and $D$, respectively.

As mentioned in [13], the computation complexity of solving the overdetermined nonlinear equations to calculate the single-bounce path delay is extremely high; regarding this, the Snell theory and the approximation algorithm are combined to transform the overdetermined nonlinear equations into linear equations, leading to considerable decrease of complexity.

As shown in Fig. 1, let A and B be the refraction points on the air-wall interface and wall-air interface, respectively. For the path $\mathrm{R}_{n} \rightarrow \mathrm{A} \rightarrow \mathrm{B}$, if the dielectric constant of the front wall is equal to the one in free-space, then the signal will transmit along the path $\mathrm{R}_{n} \rightarrow \mathrm{M} \rightarrow \mathrm{B}$, where $\mathrm{M}$ is the refraction point on the air-wall interface. In the meantime, if the dielectric constant of the front wall is assumed to be infinity large, the signal will transmit along the path $\mathrm{R}_{n} \rightarrow \mathrm{A} \rightarrow \mathrm{F}$, where $\mathrm{F}$ is the refraction point on the wall-air interface. Denote the true value of 


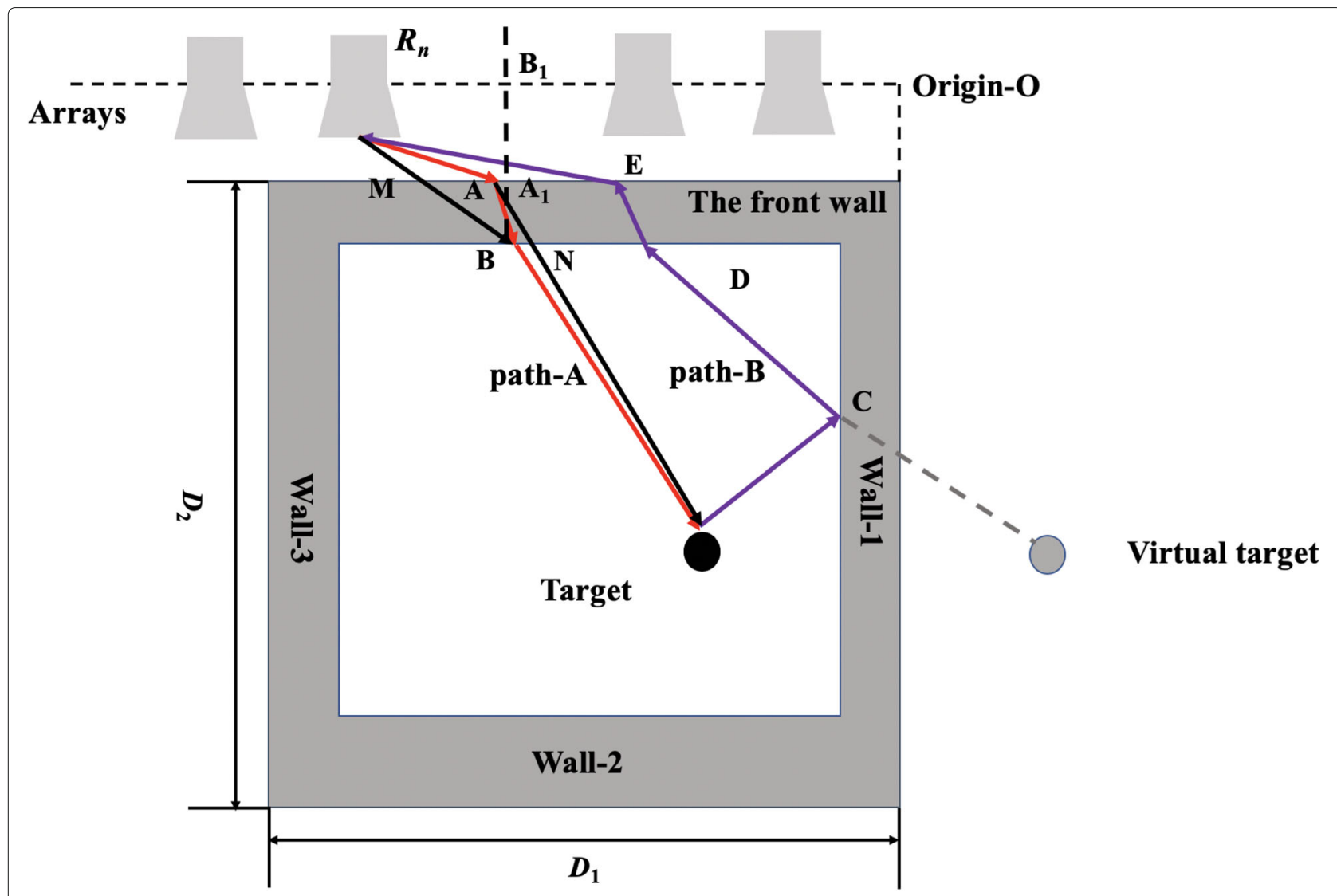

Fig. 1 Multipath propagation model

dielectric constant by $\varepsilon_{1} \in[1, \infty)$, then the $x$-axis coordinates of refraction points $A$ and $B$ satisfy the following equation:

$$
\frac{x_{\mathrm{B}}-x_{\mathrm{M}}}{x_{\mathrm{B}}-x_{\mathrm{A}}}=\sqrt{\varepsilon_{1}} .
$$

Assuming that $\mathrm{BB}_{1} \perp x$-axis with $\mathrm{A}_{1}$ being the intersection point on the air-wall interface, then the triangluars $\triangle \mathrm{R}_{n} \mathrm{BB}_{1}$ and $\triangle M B A_{1}$ are similar to each other, and according to the Triangle Similarity Law, we have

$$
\frac{x_{\mathrm{B}}-x_{n}}{d_{1}+d_{y}}=\frac{x_{\mathrm{B}}-x_{\mathrm{M}}}{d_{1}}
$$

Similarly, we can obtain the geometrical relations of the refraction points for the other propagation paths. Let $\boldsymbol{x}_{\mathrm{t}}^{\prime}=$ $\left(x_{\mathrm{t}}, y_{\mathrm{t}}\right)$ be the position of the virtual target with respect to wall 1, which is shown in Fig. 1, and can be obtained by using the Householder transformation [29]. Then, the one-way path delays for path A and path B are at the $n$th sensor position are given by

$$
\begin{aligned}
\tau_{\mathrm{A}}^{(n)}= & \frac{\sqrt{\left(x_{\mathrm{A}}-x_{n}\right)^{2}+d_{y}^{2}}+\sqrt{\varepsilon_{1}} \sqrt{\left(x_{\mathrm{B}}-x_{\mathrm{A}}\right)^{2}+d_{1}^{2}}}{c} \\
& +\frac{\sqrt{\left(x_{\mathrm{t}}-x_{\mathrm{B}}\right)^{2}+\left(y_{\mathrm{t}}-d_{1}-d_{y}\right)^{2}}}{c}, \\
\tau_{\mathrm{B}}^{(n)}= & \frac{\sqrt{\left(x_{\mathrm{E}}-x_{n}\right)^{2}+d_{y}^{2}}+\sqrt{\varepsilon_{1}} \sqrt{\left(x_{\mathrm{D}}-x_{\mathrm{E}}\right)^{2}+d_{1}^{2}}}{c} \\
& +\frac{\sqrt{\left(x_{\mathrm{t}}+x_{\mathrm{D}}\right)^{2}+\left(y_{\mathrm{t}}-d_{1}-d_{y}\right)^{2}}}{c} .
\end{aligned}
$$

Substituting (4) and (5) into (1), we obtain the received signal.

\section{Through-wall imaging with multi-path exploitation using modified Green's function}

In this section, the multi-path returned signal, which is termed as "ghosts," is exploited to further enhance the imaging performance. Similar to the conventional method where multi-path returns are not taken into account, we first derive the modified Green's function. Then, we proposed a method to identical and localize the multi-path 
returned signal from the composite data. Finally, combining with the modified Green's function, we associated and mapped the multi-path signal to obtain the image of the real target.

\subsection{Through-wall imaging based on modified Green's function}

According to traditional Green's function of three layered background mediums, the line-of-sight (LoS) propagation path in the absence of the wall is considered in the calculation, but the variation of the path propagation and phase are not considered due to the presence of the wall and thus there exists estimation error using the traditional Green's function in indoor propagation model. Considering the effect of delay inside the wall, a modified Green's function calculation model based on the non-line-of-sight (NLoS) propagation path is proposed.

By dividing the whole rectangle surrounded by walls into $N_{x} \times N_{y}$ grids, the modified Green's function at arbitrary pixel point $\boldsymbol{x}_{p}$ can be derived as [24, 25]

$$
\begin{aligned}
G\left(\boldsymbol{r}_{n}, \boldsymbol{x}_{p}, k\right)= & \frac{j}{4 \pi} \int_{-\infty}^{\infty} \frac{T\left(k_{x}\right)}{k_{1 y}} \exp \left\{j \left[k_{x}\left(x_{n}-x_{\mathrm{A}}\right)\right.\right. \\
+ & k_{1 y}\left(y_{n}-y_{\mathrm{A}}\right) \\
& +k_{2 y} d_{1}+k_{x}\left(x_{\mathrm{A}}-x_{\mathrm{B}}\right)+k_{x}\left(x_{\mathrm{B}}-x_{p}\right) \\
& \left.\left.+k_{1 y}\left(y_{n}-y_{p}\right)\right]\right\} \mathrm{d} k_{x},
\end{aligned}
$$

where $k$ is the wavenumber, $T\left(k_{x}\right)$ denotes the wall's transmission coefficient, which is calculated as

$$
T\left(k_{x}\right)=\frac{\left(1-\Gamma_{12}^{2}\right) \exp \left[j\left(k_{2 y} d_{1}-k_{1 y} d_{1}\right)\right]}{1-\Gamma_{12}^{2} \exp \left(j 2 k_{2 y} d_{1}\right)},
$$

where

$$
\begin{aligned}
k_{1 y} & =\sqrt{k_{1}^{2}-k_{x}^{2}}, \\
k_{2 y} & =\sqrt{k_{2}^{2}-k_{x}^{2}}, \\
\Gamma_{12}\left(k_{x}\right) & =\frac{k_{1 y}-k_{2 y}}{k_{1 y}+k_{2 y}},
\end{aligned}
$$

and $k_{1}$ and $k_{2}$ are the wavenumbers of freespace and the wall, respectively. Moreover, $k_{x}$ is the horizontal component of the wavenumber vector, $k_{1 y}$ and $k_{2 y}$ are the vertical components of the wavenumber vector $k_{1}$ and $k_{2}$, respectively, and $\Gamma_{12}$ is the local reflection coefficient at the air/obstacle interface. In order to solve (6) efficiently, the method of saddle point is applied here. More specifically, let

$$
\begin{aligned}
& F\left(k_{x}\right)=\frac{T\left(k_{x}\right)}{k_{1 y}}, \\
& \Phi\left(k_{x}\right)=k_{2 y} d_{1}+k_{x}\left(x_{n}-x_{p}\right)+k_{1 y}\left(y_{n}-y_{p}-d_{1}\right),
\end{aligned}
$$

then the modified Green's function can be rewritten as

$$
G\left(\boldsymbol{r}_{n}, \boldsymbol{x}_{p}, k\right)=\frac{j}{4 \pi} \int_{-\infty}^{\infty} F\left(k_{x}\right) \exp \left[j \Phi\left(k_{x}\right)\right] \mathrm{d} k_{x} .
$$

According to the stationary phase method, we have

$$
\frac{\partial \Phi\left(k_{x}\right)}{\partial k_{x}}=x_{n}-x_{p}-\frac{k_{x}}{k_{1 y}}\left(y_{n}-y_{p}\right)-\frac{k_{x}}{k_{1 y}} d_{1}+\frac{k_{x}}{k_{2 y}} d_{1}=0 .
$$

And the stationary phase point $k_{x_{0}}$ can be obtained by solving (10), given as

$$
k_{x_{0}}=\sqrt{\frac{\left(x_{\mathrm{B}}-x_{\mathrm{A}}\right)^{2} k_{2}^{2}}{d_{1}^{2}+\left(x_{\mathrm{B}}-x_{\mathrm{A}}\right)^{2}}} .
$$

Using the Taylor series expansion of $\Phi\left(k_{x}\right)$ at $k_{x}=k_{x_{0}}$ and ignoring the high order terms, the phase function $\Phi\left(k_{x}\right)$ can be approximated as

$$
\Phi\left(k_{x}\right) \approx \Phi\left(k_{x_{0}}\right)+\frac{\Phi^{\prime \prime}\left(k_{x}\right)}{2}\left(k_{x}-k_{x_{0}}\right)^{2} .
$$

Since the function $F\left(k_{x}\right)$ only has value near the stationary phase point, whereas has zero value on the other locations, the modified Green's function could be further simplified as

$$
\begin{aligned}
G\left(\boldsymbol{r}_{n}, \boldsymbol{x}_{p}, k\right) & =\frac{j}{4 \pi} \int_{-\infty}^{+\infty} F\left(k_{x}\right) \exp \left[j \Phi\left(k_{x}\right)\right] \mathrm{d} k_{x} \\
& =j F\left(k_{x_{0}}\right) \exp \left[j \Phi\left(k_{x_{0}}\right)\right] \sqrt{\frac{1}{8 \pi \mid \Phi^{\prime \prime}\left(k_{x_{0}}\right)}},
\end{aligned}
$$

where $F\left(k_{x_{0}}\right)$ is readily given by

$$
F\left(k_{x_{0}}\right)=\frac{1-\Gamma_{12}^{2}}{k_{1 y}\left(1-\Gamma_{12}^{2} \exp \left(j 2 k_{2 y} d_{1}\right)\right)} .
$$

Taking all the antennas into account, then the composite modified Green's function at $\boldsymbol{x}_{p}$ can be given as

$$
G\left(\boldsymbol{x}_{p}, k\right)=\sum_{n=1}^{N} G\left(\boldsymbol{r}_{n}, \boldsymbol{x}_{p}, k\right) .
$$

\subsection{Multi-path identification and localization}

In this subsection, a method for localizing the multi-path ghosts is described. Based on the beamforming algorithm, when $\Delta \tau_{n}\left(\boldsymbol{x}_{p}, \boldsymbol{x}_{t}\right)=\tau_{n}\left(\boldsymbol{x}_{p}\right)-\tau_{n}\left(\boldsymbol{x}_{t}\right)=0, n=1,2, \cdots, N$ holds for the $p$ th image pixel located at $x_{p}$, which depicts the locations of target or ghost in the beamformed image and the pixel $\boldsymbol{x}_{p}$ becomes focus [26]. It is noted that $\tau_{n}\left(\boldsymbol{x}_{t}\right)$ represents the two-way path delay for the direct path or the delay for the first-order multi-path at the $n$th sensor's position. The first-order ghost location is linked with $L\left(\boldsymbol{r}_{n}, \boldsymbol{x}_{t}\right)$ and $L\left(\boldsymbol{r}_{n}, \boldsymbol{x}_{t}^{\prime}\right)$, where $L\left(\boldsymbol{r}_{n}, \boldsymbol{x}_{t}\right)$ and $L\left(\boldsymbol{r}_{n}, \boldsymbol{x}_{t}^{\prime}\right)$ are the single-bounce propagation distances of path A and path 
B, respectively. Consider the ghost located at $\boldsymbol{x}_{p}$, which satisfies the following equation

$$
L\left(\boldsymbol{x}_{n}, \boldsymbol{x}_{p}\right)=\frac{L\left(\boldsymbol{r}_{n}, \boldsymbol{x}_{t}\right)+L\left(\boldsymbol{r}_{n}, \boldsymbol{x}_{t}^{\prime}\right)}{2} .
$$

The first-order ghost location is relatively fixed in the original beamformed image. We can determine the travel distance from the $n$th antenna to the pixel along the direct or indirect way, which indicates that $L\left(\boldsymbol{x}_{n}, \boldsymbol{x}_{p}\right)$ is a known parameter. If we provide a circular contour with radius $L\left(\boldsymbol{r}_{n}, \boldsymbol{x}_{p}\right)$ centered at $\mathrm{R}_{n}$, the ghost must lie on it. Similarly, Fig. 2 shows that using two circular contours with the size of $L\left(\boldsymbol{r}_{n}, \boldsymbol{x}_{p}\right)$ by $L\left(\boldsymbol{r}_{m}, \boldsymbol{x}_{p}\right)$ centered at $\mathrm{R}_{n}$ and $\mathrm{R}_{m}$, respectively, the ghost must appear in the place where the circular contours cross. Nevertheless, two positions, which are crossed by the contours, are presented: one is in front of the array and the other is behind it. Because of the imaging scene locate directly in front of the array, it is reasonable to suppose that the location of the ghost is in front of it. The equations for both contours can be interpreted by

$$
\begin{aligned}
& \left(x+x_{n}\right)^{2}+y^{2}=L^{2}\left(\boldsymbol{r}_{n}, \boldsymbol{x}_{p}\right), \\
& \left(x+x_{m}\right)^{2}+y^{2}=L^{2}\left(\boldsymbol{r}_{m}, \boldsymbol{x}_{p}\right) .
\end{aligned}
$$

In the real-world radar system, the array contains $N$ elements. Then, there exist $\left\lfloor\frac{N}{2}\right\rfloor$ possible pairs if each element is considered only once. Providing two circular contours with the size of $L\left(\boldsymbol{r}_{n}, \boldsymbol{x}_{p}\right)$ and $L\left(\boldsymbol{r}_{n+\left\lfloor\frac{N}{2}\right\rfloor}, \boldsymbol{x}_{p}\right)$ which are centered at $\mathrm{R}_{n}$ and $\mathrm{R}_{n+\left\lfloor\frac{N}{2}\right\rfloor}$ respectively, the procedure for identifying the ghost locations $\boldsymbol{x}_{p n}$ is as follows.

For all $n=1, \cdots,\left\lfloor\frac{N}{2}\right\rfloor$, we obtain the intersection points $\boldsymbol{x}_{p n}$ of the two circular contours by letting their analytical expression be equal. Then, taking expectation with respect to $n$, we have the final value of ghost location as per

$$
\boldsymbol{x}_{p}=\frac{1}{\left\lfloor\frac{N}{2}\right\rfloor} \sum_{n=1}^{\left\lfloor\frac{N}{2}\right\rfloor} \boldsymbol{x}_{p n} .
$$

For the ghosts associated with walls 2 and 3, the location of them can be obtained in a similar way.

\subsection{Multi-path association and mapping}

After we localize the multi-path ghosts, we can then associate and map these signals back to the position of the real target. To enhance the signal-to-clutter ratio and remove the false targets, a composite image can be constructed by using Hadamard product of back propagation (BP) image and modified Green's function as follows:

$$
\mathbf{I}_{G}(\cdot)=\mathbf{I}^{\mathrm{BP}}(\cdot) \bigotimes \mathbf{G}(\cdot)
$$

where $\mathbf{I}^{\mathrm{BP}}(\cdot)$ is Back Projection imaging matrix [10], $\mathbf{G}(\cdot) \in \mathcal{C}^{N_{x} \times N_{y}}$ is the modified Green's function matrix, whose element for arbitrary pixel is obtained in

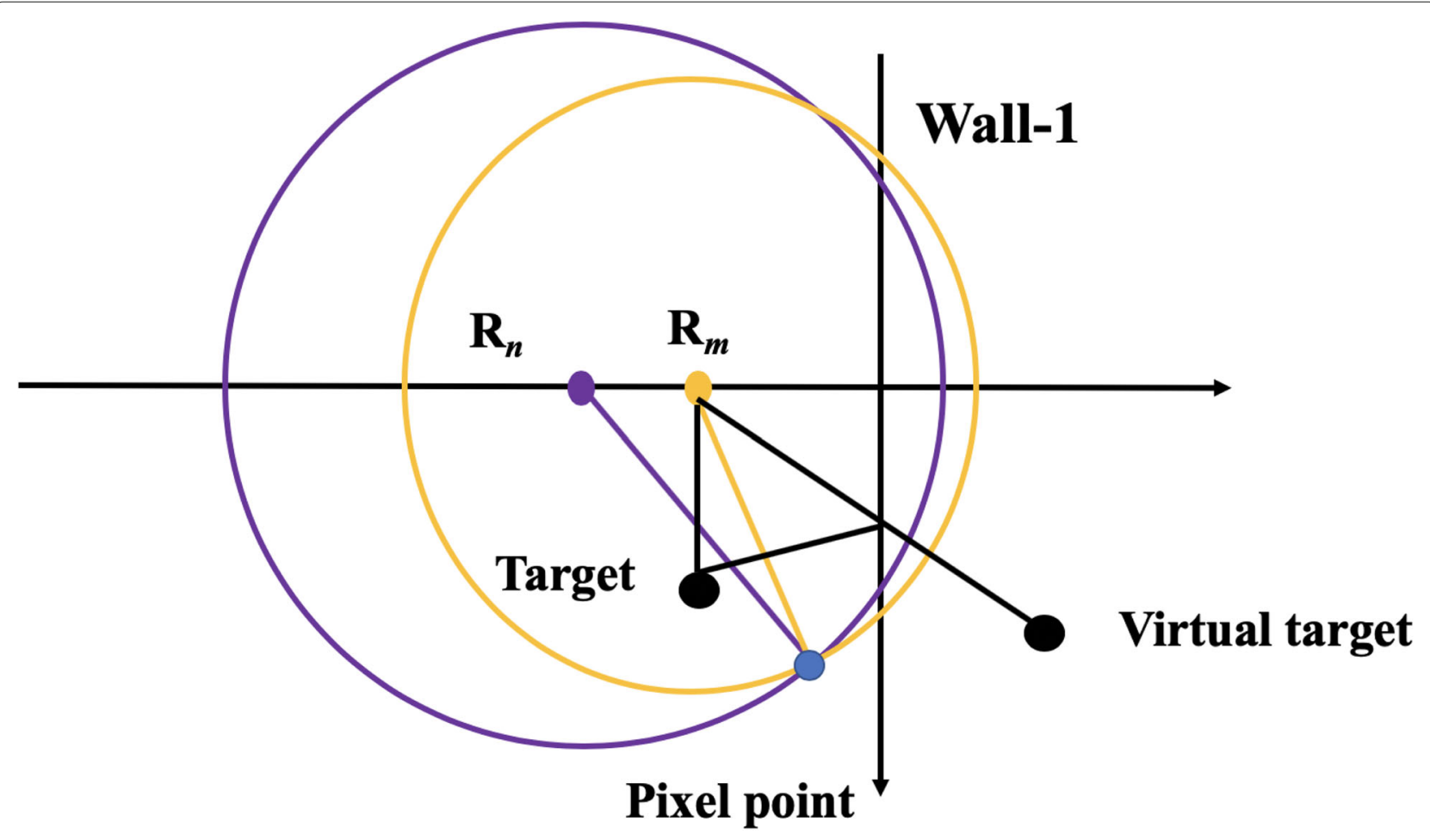

Fig. 2 Location of multi-path ghost 
Section 3.1, and $\otimes$ denotes the Hadamard product operation. Combining the strong shadow region of the target with the multi-path ghost associated with wall 2 , the image $\mathbf{I}^{\mathrm{BP}}(\cdot)$ has a strong false target at the same crossrange as the target and at a downrange position equal to the back wall. The target location can be identified under the simple threshold operation.

With regard to the focused multi-path ghosts, the association and mapping of them are considered. Let $\mathcal{X}$ and $\mathcal{Y}$ be the index of the crossrange and downrange, respectively, and we define the two-dimensional space as $\mathcal{W}=$ $\mathcal{X} \times \mathcal{Y}$. The region we are interested in, $\mathbf{I}_{G}(\cdot)$, consists of $N_{x} \times N_{y}$ grids, where the pixel is located at the center of each grid. Without loss of generality, we assume that the target is located at $x=(x, y) \in \mathcal{W}$, and we denote the focused multi-path ghost from walls 1,2 , and 3 as $\boldsymbol{x}_{k}^{\text {wall }}=\left(x_{k}^{\text {wall }}, y_{k}^{\text {wall }}\right), k=1,2,3,\left(x_{k}^{\text {wall }}, y_{k}^{\text {wall }}\right) \in \mathcal{W}$, respectively. Then, the composite image values at the target can be represented in a $N_{x} \times N_{y}$ complexed-value matrix form as $\mathbf{I}_{G}^{x}(\cdot) \in \mathcal{C}^{N_{x} \times N_{y}}$ with the elements being $I_{G}\left(\boldsymbol{x}_{p} ; \boldsymbol{x}\right)$. Similarly, the composite image values are evaluated at $\boldsymbol{x}_{k}^{\text {wall }}, k=1,2,3 \in \mathcal{W}$ and are denoted as the matrices $\mathbf{I}_{G k}^{\text {wall }}(\cdot) \in \mathcal{C}^{N_{x} \times N_{y}}$ with the elements being $I_{G}\left(\boldsymbol{x}_{p} ; \boldsymbol{x}_{k}^{\text {wall }}\right)$. To prevent the differences in intensity, the images are normalized such that the elements of these matrices are within the interval $[0,1]$. For the composite image matrix elements, a threshold operation is considered, with the threshold $\beta_{\text {th }}$, i.e.,

$$
\widehat{\mathbf{I}}(x, y)=\left\{\begin{array}{l}
1, I(x, y)>\beta_{\mathrm{th}} \\
0, \text { otherwise }
\end{array}\right.
$$

where $I(x, y)$ is either $I_{G k}^{\text {wall }}(x, y)$ or $I_{G}^{x}(x, y)$, and $\widehat{\mathbf{I}}_{G k}^{\text {wall }}(\cdot)$ contains many peak values in and near the locations of the ghosts and $\hat{\mathbf{I}}_{G}^{x}(\cdot)$ contains many peaks value in the vicinity of the target location. Moreover, we define $\tilde{\mathbf{I}}_{G}^{x}(\cdot)=$ $\mathbf{I}-\hat{\mathbf{I}}_{G}^{x}(\cdot)$, where $\mathbf{I}$ is the identity matrix. If the pixel $\boldsymbol{x}_{p}$ is located at the location of the focused multi-path ghost, $\mathbf{I}^{\mathrm{BP}}\left(x_{k}^{\text {wall }}, y_{k}^{\text {wall }}\right)$ is assumed to be a large value. In that case, $\tilde{\mathbf{I}}_{G}^{x}(\cdot)$ can be used to alleviate the multi-path pixels that are very close to the genuine target if they are at position $x_{k}^{\text {wall }}$. It can also be used to reject the pixel location being in the vicinity of the true target location. In order to associate and map the ghost, an association matrix $\mathbf{I}_{\text {inter }}$ can be constructed, and the element of $\mathbf{I}_{\text {inter }}$ is calculated as

$I_{\text {inter }}\left(\boldsymbol{x}_{p}\right)=\sum_{\boldsymbol{x}_{p} \in \mathcal{W}}\left|\mathbf{I}^{\mathrm{BP}} \bigotimes\left(\mathbf{I}-\hat{\mathbf{I}}_{G}^{\boldsymbol{x}_{p}}+\hat{\mathbf{I}}_{G 1}^{\text {wall }}+\hat{\mathbf{I}}_{G 2}^{\text {wall }}+\hat{\mathbf{I}}_{G 3}^{\text {wall }}\right)\right|$.

Repeating (25) for all possible pixel locations, matrix $\mathbf{I}_{\text {inter }} \in \mathcal{C}^{N_{x} \times N_{y}}$ can be obtained. Assuming deep nulls at the region of ghost and the strongest peak at the region of target, the final image of the multi-path exploitation is obtained by

$$
\mathbf{I}_{\text {final }}=\mathbf{I}_{\mathrm{f}} \bigotimes \mathbf{I}_{\text {inter }} \bigotimes \mathbf{I}^{\mathrm{BP}},
$$

where the element of $\mathbf{I}_{\mathrm{f}}$ is calculated as

$$
I_{\mathrm{f}}(\boldsymbol{x})=\left\{\begin{array}{l}
1, \frac{I_{\text {inter }}(\boldsymbol{x})}{\max I_{\text {inter }}(\boldsymbol{x})}>\lambda_{\text {th }}, \\
0, \text { otherwise, }
\end{array}\right.
$$

and $\lambda_{\text {th }} \in[0,1]$ is a predefined threshold.

\section{Results and discussions}

In this section, numerical simulations are given to evaluate the effectiveness of the proposed modified Green's function. The reference scenario and measurement configuration are best defined in the preceding section. The image domain is $x=2.0 \mathrm{~m}$ and $y=1.48 \mathrm{~m}$ in width and length, respectively. The transceiver array scans the region of interest from the distance between the front $0.3 \mathrm{~m}$ from the front wall, along a line parallel to the wall in $x$ direction from $0 \mathrm{~m}$ to $-2 \mathrm{~m}$ with a step of $0.05 \mathrm{~m}$. The accurate scattered field is calculated in time domain by gprMax software, we define the relative permittivity, conductivity, and thickness are $\varepsilon_{1}=6.0, \sigma_{1}=0.01 \mathrm{~S} / \mathrm{m}$, and $d_{1}=0.1$ $\mathrm{m}$ for the front wall in the software, respectively. For a line source, its radiates Ricker wavelet at $900 \mathrm{MHz}$ center frequency and with a bandwidth of $900 \mathrm{MHz}$. The intensities of the image are described in $\mathrm{dB}$, unless noted otherwise.

\subsection{Single target}

The numerical results for the imaging of one target behind a homogeneous wall are presented in this subsection and the square target whose size is $0.04 \mathrm{~m}$ is centered at $(-0.9,0.75) \mathrm{m}$. The composite image in Fig. 3 is obtained through Hadamard operation.Since the reflection and transmission coefficients for single-bounce trip along with path $B$, path $C$, and path $D$ are smaller than that along with path $\mathrm{A}$, the pixel values at the region of ghosts are significantly smaller than that at the region of target. Then, the target location and the focused multi-path ghosts' locations can be identified by the threshold operation. Figure 4 is an intermediate image; it shows the existing deep nulls at the locations of ghosts and the strongest peak can identify the location of the target. Figure 5 shows the result after association and mapping. This figure shows the target and the ghosts that have been mapped back to the target location. Since the method of background subtraction can not filter out the interference produced by the target, the extraneous target is retained, which is located at $(-0.9,0.46) \mathrm{m}$.

\subsection{Multiple targets}

The configuration of radar system is equal to the previous case. One target is located at $(-0.7,0.75) \mathrm{m}$ and the other at $(-1.25,0.65) \mathrm{m}$. As shown in Fig. 6, it reveals not only the original beamforming image, but some false targets are also shown from the figure. This is probably due 


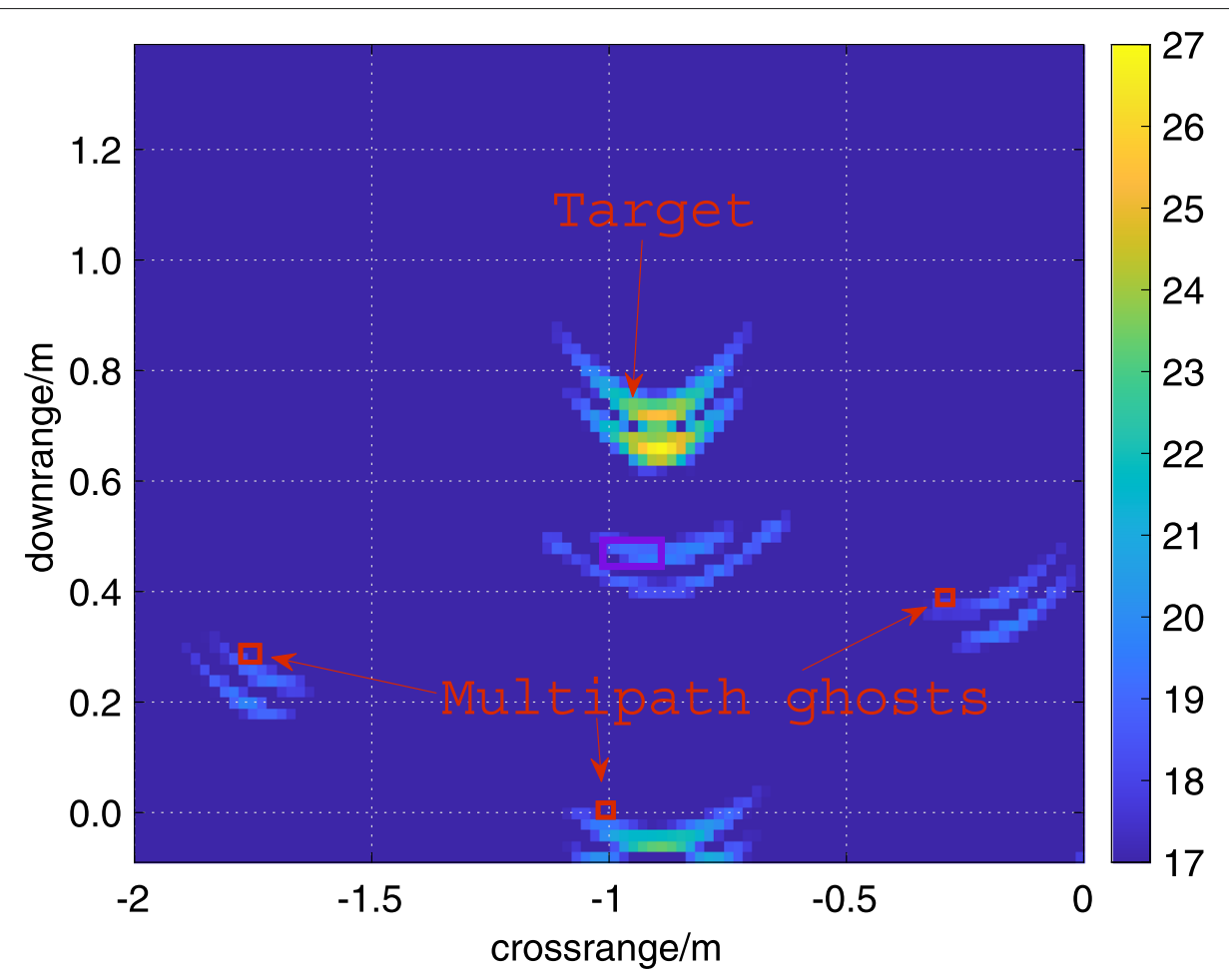

Fig. 3 Single target case: composite image

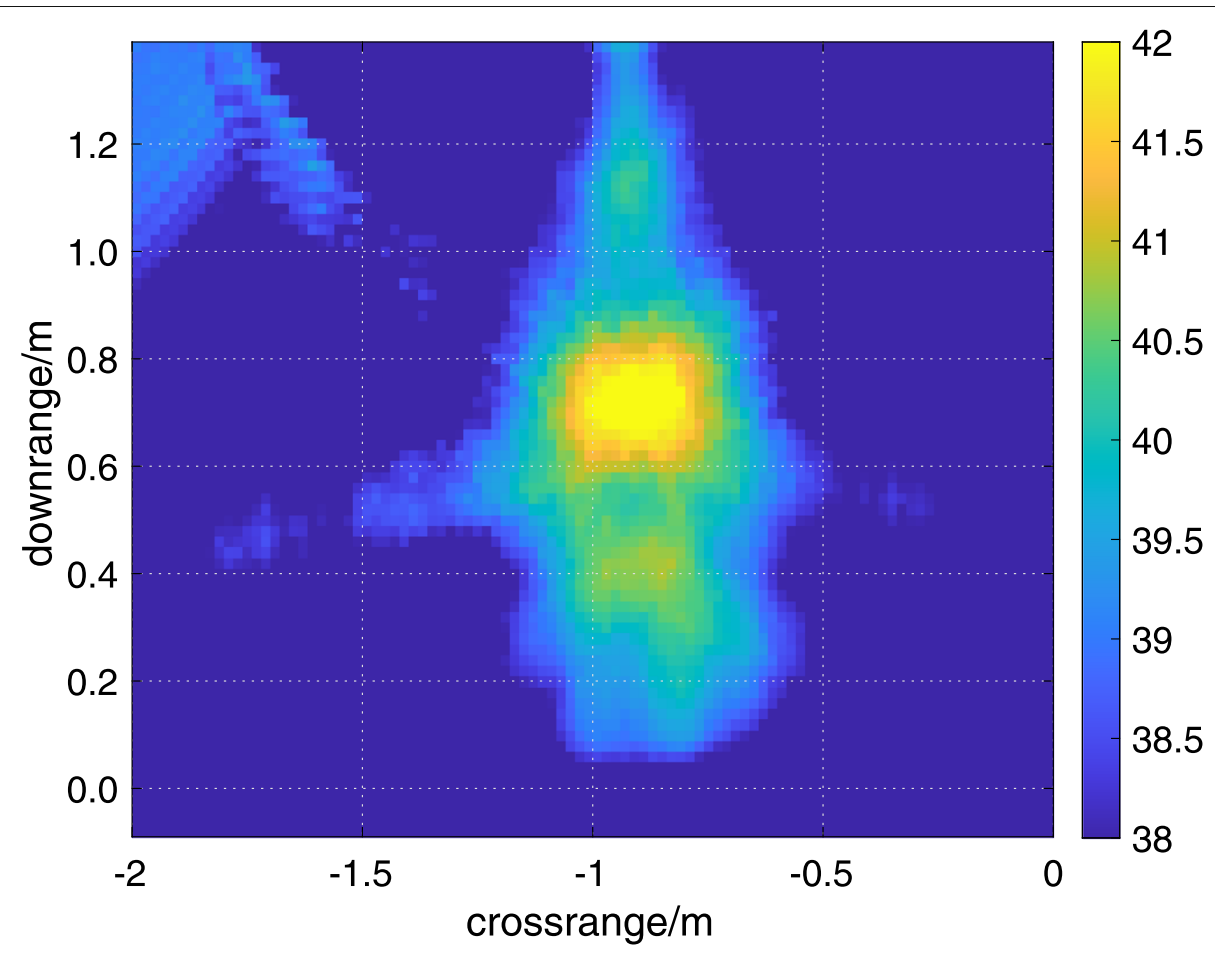

Fig. 4 Single target case: intermediate image 

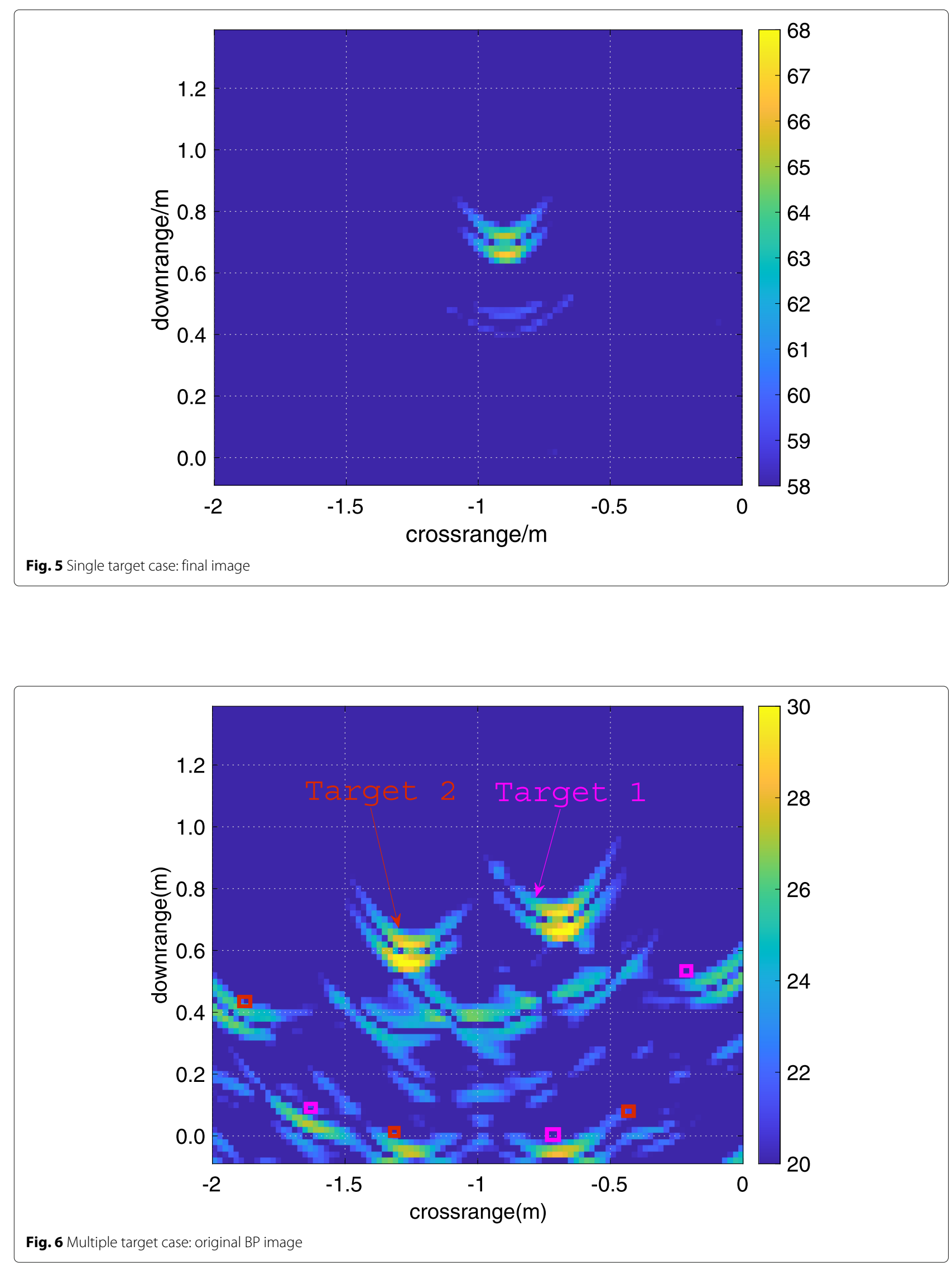


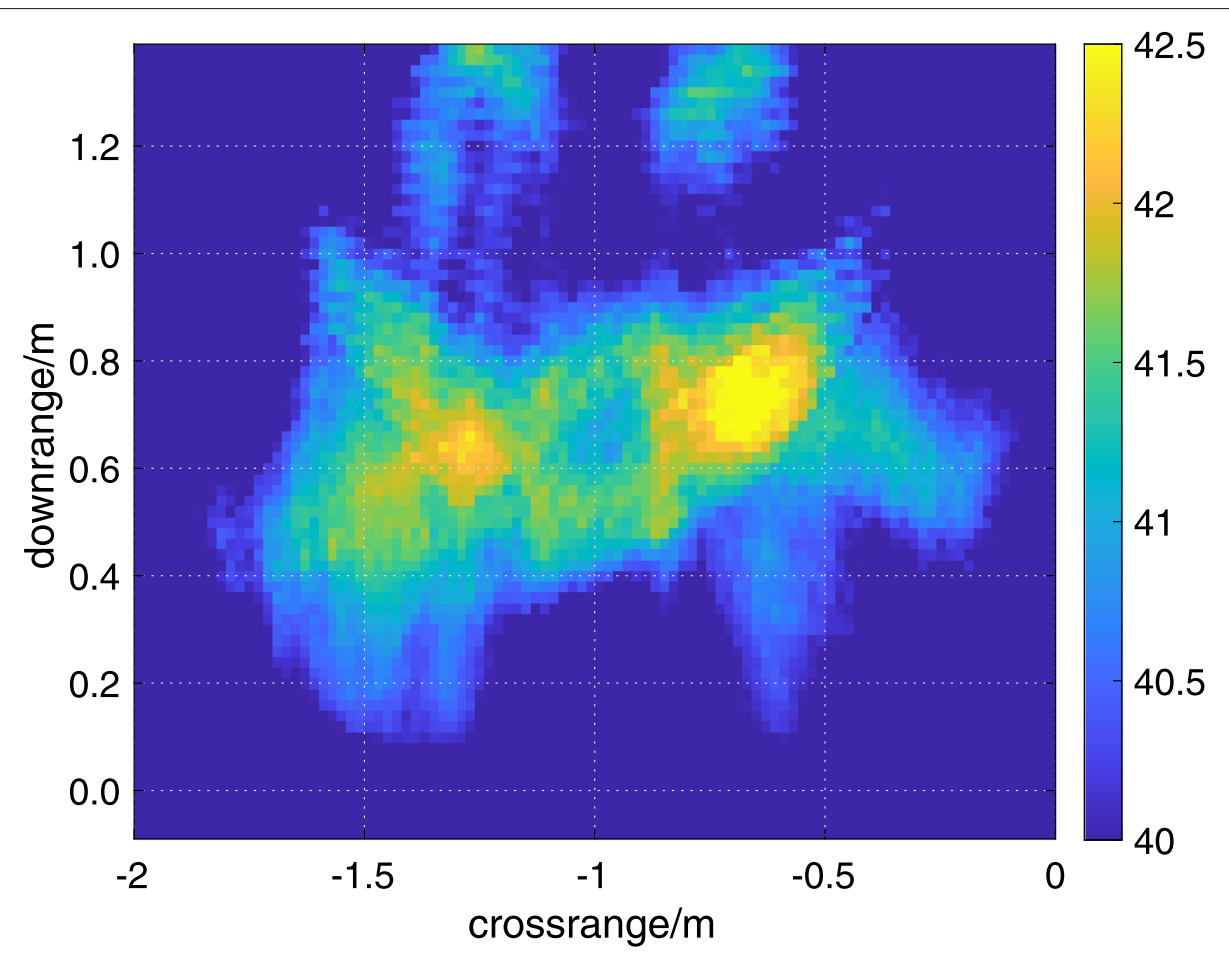

Fig. 7 Multiple target case: intermediate image

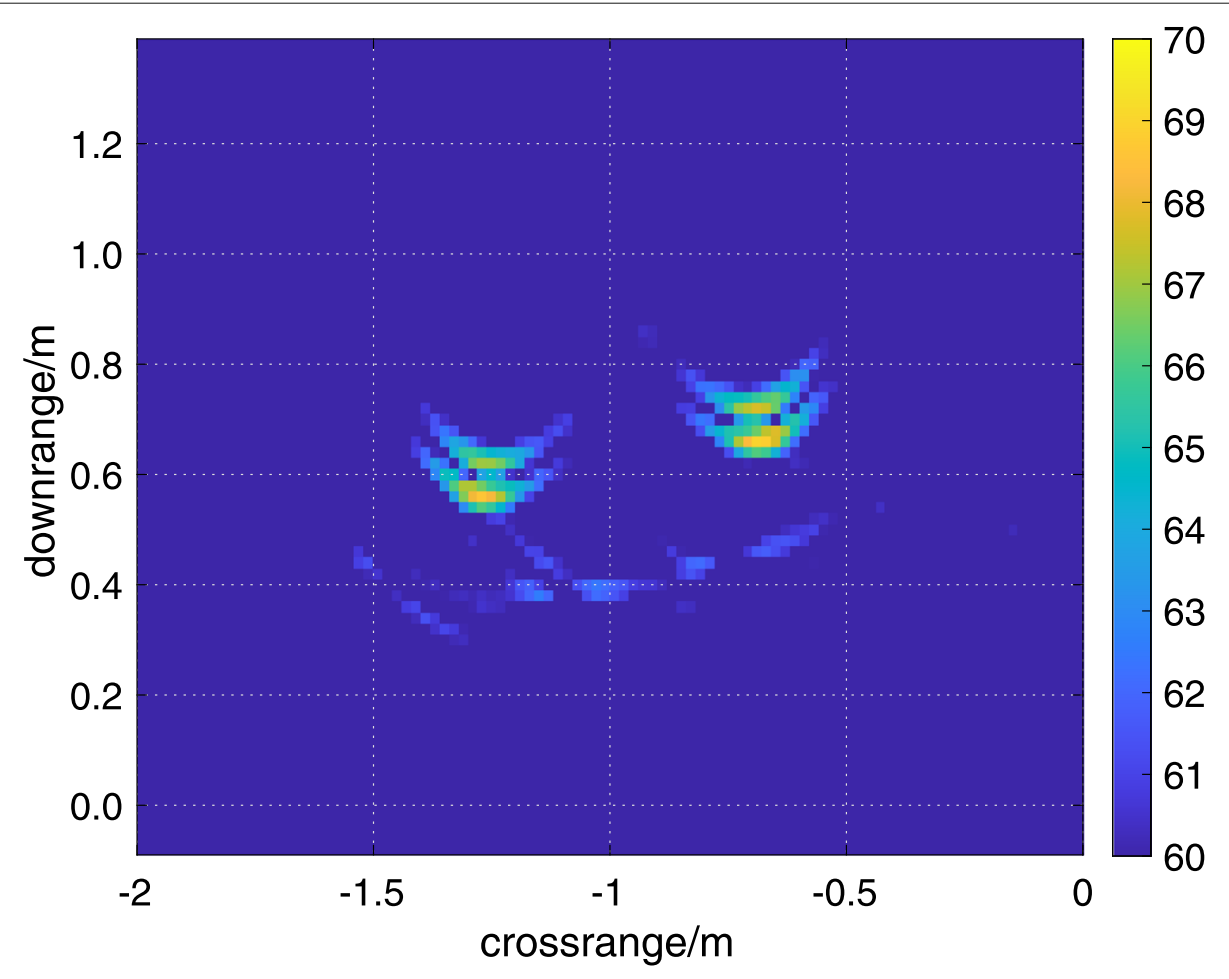

Fig. 8 Multiple target case: final image 
Table 1 The simulations for the SCR cases

\begin{tabular}{llllll}
\hline & \multicolumn{2}{l}{ SCR single } & & & \multicolumn{2}{l}{ SCR multiple } & \\
\cline { 2 - 3 } & Before & After & & Before & After \\
\hline$[7]$ & -6.6878 & 3.2844 & & -5.0952 & 1.6054 \\
This paper & -6.6878 & 6.8612 & & -5.0952 & 4.4013 \\
\hline
\end{tabular}

to the interactions with the wall or the targets themselves. It is noted that proposed algorithm can not eliminate erroneous targets. The intermediate image is shown in Fig. 7, which are existing deep nulls at the regions of ghosts and the strongest peak at the locations of targets. In Fig. 8, it reveals the result of association and mapping. After exploitation, we can distinctly see two targets and map ghosts back to real targets. Figure 8 shows the compound interactions between the walls and targets, leading to some remnants persisting.

As noise was not taken into account in our scenario, we consider the signal SCR, defined as the ratio of the power in the target regions to the power in the rest of the image as the performance metric [27]. Through multipath exploitation, the case of the SCR about single target and multiple targets are reported in Table 1 by using the method in [7] and the proposed method. It can be seen that both methods can enhance the SCR after the procedure. According to the exploitation procedure, for single target case, the SCR increases by about $9.9722 \mathrm{~dB}$ at the location of the target by using the method in [7] and about $13.549 \mathrm{~dB}$ growth by the proposed method. For multiple targets case, the SCR component is $9.4965 \mathrm{~dB}$ and $6.7006 \mathrm{~dB}$ for the proposed method and the method in [7], respectively.

\subsection{Results of real measured dataset}

An experimental dataset comprising of a single target in an enclosed structure is collected to demonstrate the improvement from multi-path exploitation. A throughwall radar system is used for signal synthesis and data collection. The system is operated at a central frequency of $800 \mathrm{MHz}$. The human is stationary in the imaging scene and the standoff distance to the back side of the front wall is $1.4 \mathrm{~m}$. The size of the imaging scene is $4.0 \times 3.2 \mathrm{~m}^{2}$, consisting of $125 \times 100$ grids and the size of each grid is $0.032 \times 0.032 \mathrm{~m}^{2}$. Assuming that the walls are singlelayered and homogeneous with permittivity $\varepsilon_{1}=6.0$ and thickness $d_{1}=0.1 \mathrm{~m}$, which can be estimated through the approach proposed in [28].

Figure 9 shows the raw radar data with heavy clutter in the time domain. Due to the complex imaged environment, the electromagnetic interactions among the targets, the unidentified walls, and the furnitures distort the received signals, leading to severe interference to the

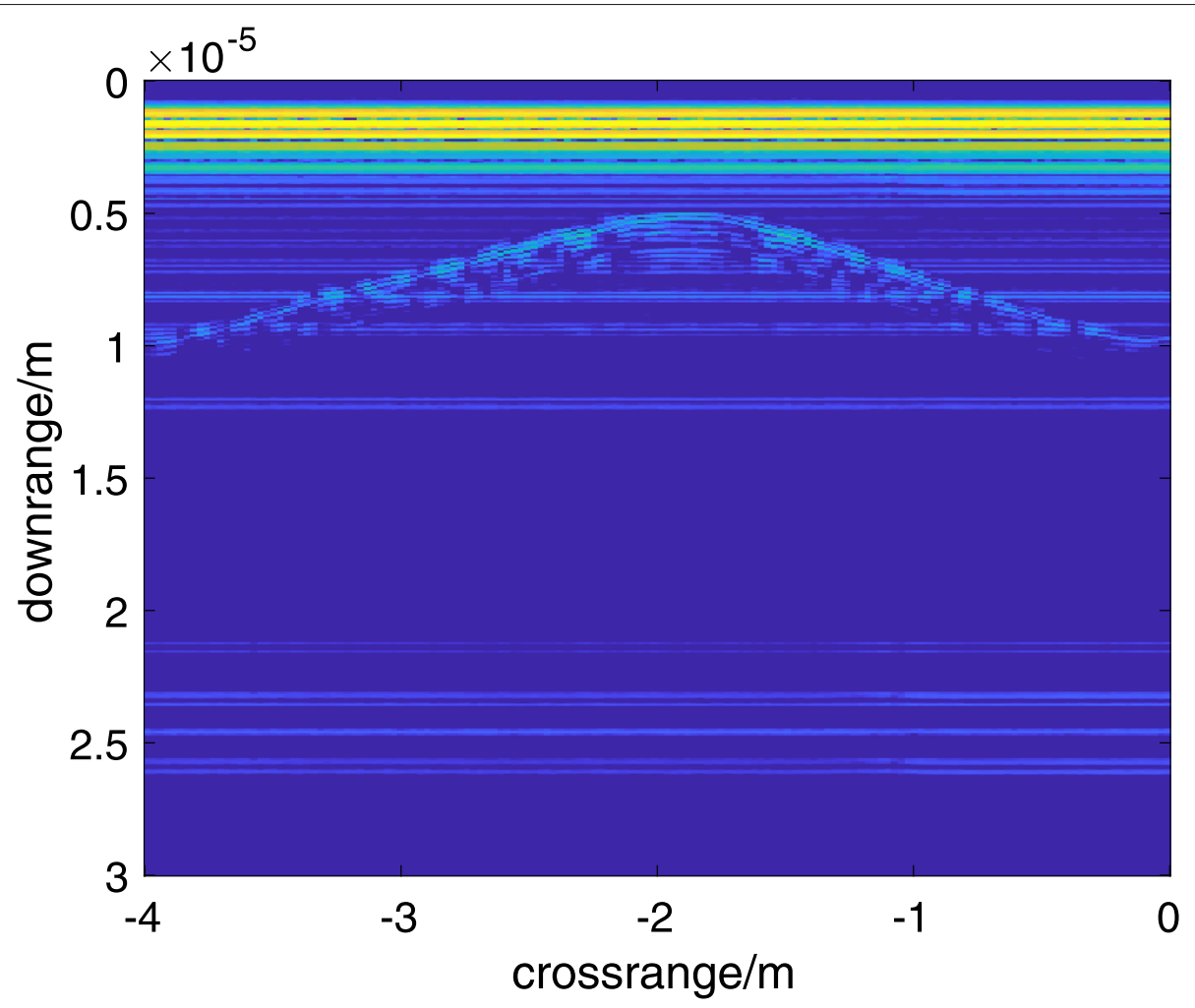

Fig. 9 Measured data: raw radar data 


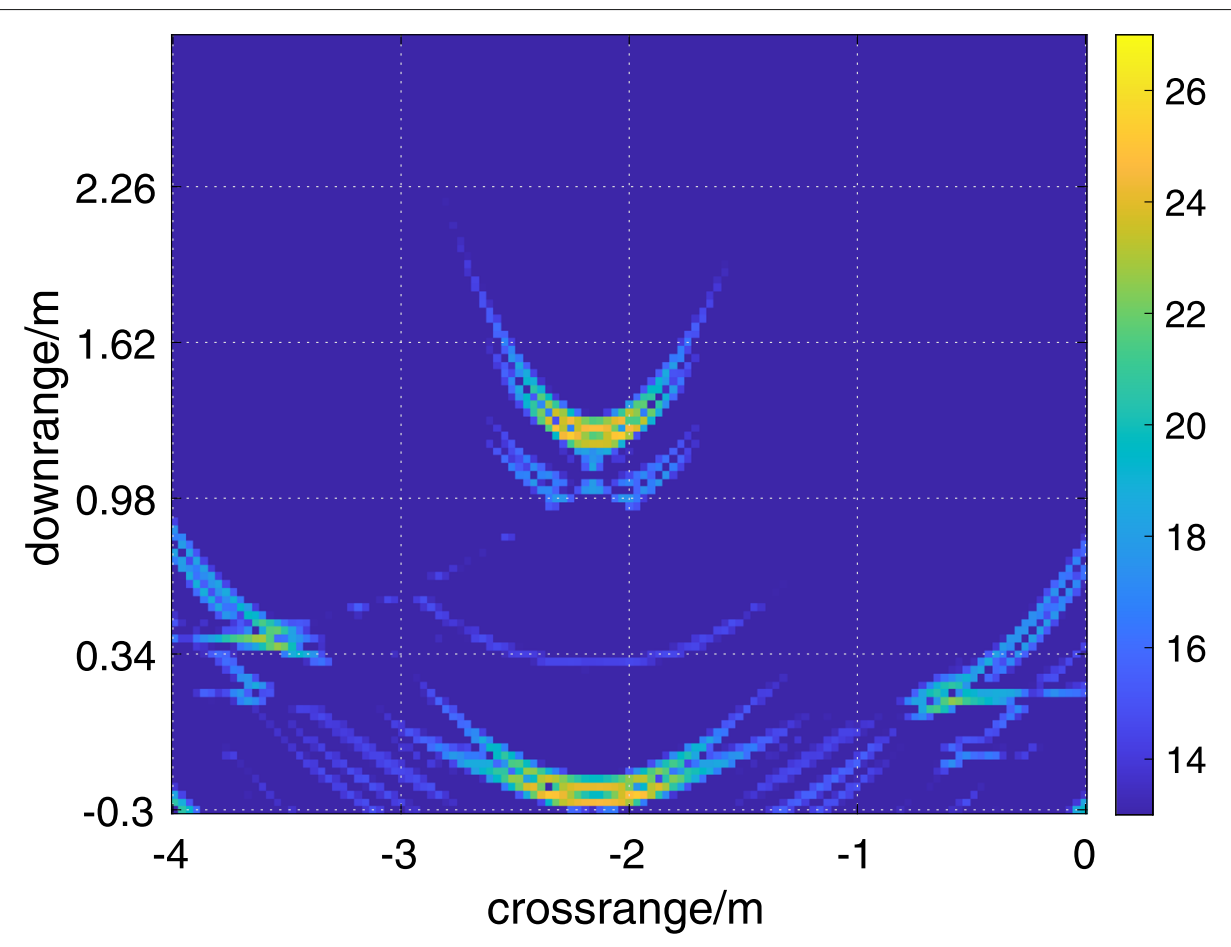

Fig. 10 Measured data: original BP image

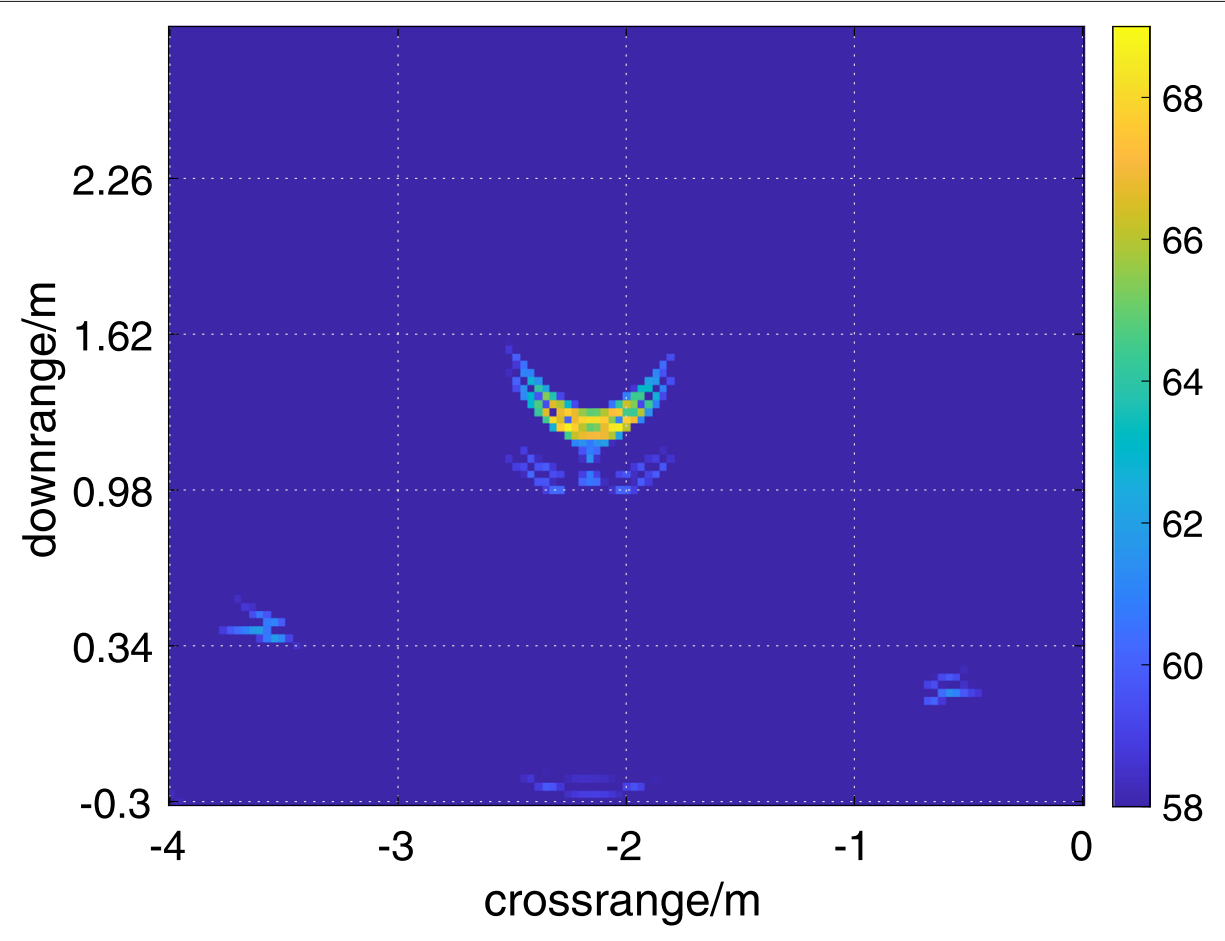

Fig. 11 Measured data: results after exploitation 
exploitation results. Compared with the curve presented by target, the multi-path returns are relatively weak due to multiple reflections occurring. Using BP imaging algorithm, the result is shown in Fig. 10. There exist so many ghosts or false targets that the location of the target can not be identified with a simple operation. Figure 11 is the result of association and mapping; the majority of ghosts are removed, and we can ensure the target location.

\section{Conclusion}

In this paper, a modified Green's function-based method is proposed to remove the focused multi-path ghosts and enhance the SCR by exploiting multi-path returned signals on the basis of the knowledge of geometric model. An algorithm combining the modified Green's function of through-wall radar with the algorithm of BP imaging can be built, which associates and maps the ghosts to their real target. Simulation and real radar experiment results confirm that the proposed method can strengthen the quality of TWRI and enhance SCR at the target location.

\section{Abbreviations}

BP: Back Projection; LoS: Line-of-sight; MIMO: Multiple-input multiple-output; NLoS: Non-line-of-sight; SCR: Signal-to-clutter ratio; TWRI: Through-wall radar imaging; UWB: Ultra-wide band

\section{Authors' contributions}

SW proposed the algorithm and developed the mathematical derivations as well as the majority of simulation results. $\mathrm{HZ}$ helped to check the simulation codes and supported the real data measurements. The paper was written by $\mathrm{SW}$ and revised by $\mathrm{HZ}$ and $\mathrm{RD}$. After receiving the comments of reviewer and editor, SL helped to revise the paper. All authors read and approved the final manuscript.

\section{Funding}

The research in this article is supported by the National Natural Science Foundation of China (No.61561034, No.61261010, and No.71461021) and the Natural Science Foundation of Jiangxi Province (2015BA B207001).

\section{Availability of data and materials}

Please contact the authors for data requests.

\section{Competing interests}

The authors declare that they have no competing interests.

\section{Author details}

${ }^{1}$ School of Sciences, Nanchang University, 330031 Nanchang, People's Republic of China. ${ }^{2}$ School of Information and Engineering, Nanchang University, 330031 Nanchang, People's Republic of China.

Received: 11 October 2019 Accepted: 31 December 2019

Published online: 12 February 2020

\section{References}

1. C. H. Seng, A. Bouzerdoum, M. G. Amin, S. L. Phung, Two-stage fuzzy fusion with applications to through-the-wall radar imaging. IEEE Geosci. Remote Sens. Lett. 10, 687-691 (2013). https://doi.org/10.1109/LGRS. 2012.2218570

2. L. M. Frazier, Surveillance through walls and other opaque materials. IEEE Aerosp. Electron. Syst. Mag. 11, 6-9 (1996). https://doi.org/10.1109/62. 538794

3. X. Wang, G. Li, Q. Wan, R. J. Burkholder, Look-ahead hybrid matching pursuit for multipolarization through-wall radar imaging. IEEE Trans.
Geosci. Remote Sens. 55, 4072-4081 (2017). https://doi.org/10.1109/ TGRS.2017.2687478

4. X. Liu, H. Leung, G. A. Lampropoulos, Effect of wall parameters on ultra-wideband synthetic aperture through-the-wall radar imaging. IEEE Trans. Aerosp. Electron. Syst. 48, 3435-3449 (2012). https://doi.org/10. 1109/TAES.2012.6324724

5. L. Wang, B. Yazici, Bistatic synthetic aperture radar imaging using ultranarrowband continuous waveforms. IEEE Trans. Image Process. 21, 3673-3686 (2012). https://doi.org/10.1109/TIP.2012.2193134

6. H. Ma, T. W. C. Brown, A. T. S. Ho, UWB reactive near field detection for smart packages: a comprehensive study using eggs. IEEE Trans. Antennas Propag. 65, 3691-3701 (2017). https://doi.org/10.1109/TAP.2017.2705020

7. P. Setlur, M. Amin, F. Ahmad, Multipath model and exploitation in through-the-wall and urban radar sensing. IEEE Trans. Geosci. Remote Sens. 49, 4021-4034 (2011)

8. M. G. Amin, F. Ahmad, Wideband synthetic aperture beamforming for through-the-wall imaging. IEEE Signal Process. Mag. 25, 110-113 (2008). https://doi.org/10.1109/MSP.2008.923510

9. E. J. Baranoski, Through-wall imaging: historical perspective and future directions. J. Franklin Inst. 345, 556-569 (2008). https://doi.org/10.1016/j. jfranklin.2008.01.005

10. P. C. Chang, R. J. Burkholder, J. L. Volakis, Adaptive CLEAN with target refocusing for through-wall image improvement. IEEE Trans Antennas Propag. 58, 155-162 (2010). https://doi.org/10.1109/TAP.2009.2036131

11. W. Zhang, A. Hoorfar, C. Thajudeen, F. Ahmad, Full polarimetric beamforming algorithm for through-the-wall radar imaging. Radio Sci. 46, 1-17 (2011). https://doi.org/10.1029/2010RS004631

12. F. Ahamd, M. G. Amin, Multi-location wideband synthetic aperture imaging for urban sensing applications. J. Franklin Inst. 345, 618-639 (2008). https://doi.org/10.1016/j.jfranklin.2008.03.003

13. M. Leigsnering, F. Ahmad, M. Amin, A. Zoubir, Multipath exploitation in through-the-wall radar imaging using sparse reconstruction. IEEE Trans Aerosp. Electron. Syst. 50, 920-939 (2014). https://doi.org/10.1109/TAES 2013.120528

14. M. Leigsnering, F. Ahmad, M. G. Amin, A. M. Zoubir, Compressive sensing-based multipath exploitation for stationary and moving indoor target localization. IEEE Sel. Topics Signal Process. 9, 1469-1483 (2015). https://doi.org/10.1109/JSTSP.2015.2464177

15. X. Lan, A. J. Ma, P. C. Yuen, R. Chellappa, Joint sparse representation and robust feature-level fusion for multi-cue visual tracking. IEEE Trans. Image Process. 24, 5826-5841 (2015). https://doi.org/10.1109/TIP.2017.2777183

16. X. Lan, S. Zhang, P. C. Yuen, R. Chellappa, Learning common and feature-specific patterns: a novel multiple-sparse-representation-based tracker. IEEE Trans. Image Process. 27, 2022-2037 (2018). https://doi.org/ 10.1109/TIP.2015.2481325

17. X. Lan, M. Ye, R. Shao, P. C. Yuen, H. Zhou, Learning modality-consistency feature templates: a robust RGB-infrared tracking system. IEEE Trans. Ind. Electron. 24, 9887-9897 (2019). https://doi.org/10.1109/TIE.2019.2898618

18. X. Lan, M. Ye, R. Shao, B. Zhong, D. K. Jain, H. Zhou, Online non-negative multi-modality feature template learning for RGB-assisted infrared tracking. IEEE Access. 7, 67761-67771 (2019). https://doi.org/10.1109/ ACCESS.2019.2916895

19. X. Lan, M. Ye, S. Zhang, H. Zhou, P. C. Yuen, Modality-correlation-aware sparse representation for RGB-infrared object tracking. Pattern Recog. Lette (2018). https://doi.org/10.1016/j.patrec.2018.10.002

20. J. Liu, G. Cui, Y. Jia, L. Kong, X. Yang, Sidewall detection using multipath in through-wall radar moving target tracking. IEEE Geosci. Remote Sens. Lett. 12, 1372-1376 (2015). https://doi.org/10.1109/LGRS.2015.2403133

21. S. Guo, G. Cui, L. Kong, X. Yang, An imaging dictionary based multipath suppression algorithm for through-wall radar imaging. IEEE Trans Aerosp. Electron. Syst. 54, 269-283 (2018). https://doi.org/10.1109/TAES.2017. 2756298

22. S. Z. Gurbuz, W. L. Melvin, D. B. Williams, A nonlinear-phase model-based human detector for radar. IEEE Trans Aerosp. Electron. Syst. 47, 2502-2513 (2011). https://doi.org/10.1109/TAES.2011.6034647

23. S. Z. Gurbuz, W. L. Melvin, D. B. Williams, Kinematic model-based human detectors for multi-channel radar. IEEE Trans Aerosp. Electron. Syst. 48, 1306-1318 (2012). https://doi.org/10.1109/TAES.2012.6178063

24. F. Soldovieri, R. Solimene, Through-wall imaging via a linear inverse scattering algorithm. IEEE Geosci. Remote Sens. Lett. 4, 513-517 (2007). https://doi.org/10.1109/LGRS.2007.900735 
25. I. Catapano, L. Crocco, A qualitative inverse scattering method for through-the-wall imaging. IEEE Geosci. Remote Sens. Lett. 7, 685-689 (2010). https://doi.org/10.1109/LGRS.2010.2045473

26. G. E. Smith, B. G. Mobasseri, Analysis and exploitation of multipath ghosts in radar target image classification. IEEE Trans. Image. Process. 23, 1581-1592 (2014). https://doi.org/10.1109/TIP.2014.2302683

27. Y.-S. Yoon, M. Amin, Spatial filtering for wall-clutter mitigation in through-the-wall radar imaging. IEEE Trans. Geosci. Remote Sens. 47, 2192-3208 (2011). https://doi.org/10.1109/TGRS.2009.2019728

28. T. Jin, B. Chen, Z. Zhou, Image-Domain estimation of wall parameters for autofocusing of through-the-wall SAR imagery. IEEE Trans. Geosci. Remote Sens. 51, 1836-1843 (2013). https://doi.org/10.1109/TGRS.2012.2206395

29. N. K. Tsao, A note on implementing the Householder transformation. SIAM J. Numer. Anal. 12, 53-58 (1975). https://doi.org/10.1137/0712005

\section{Publisher's Note}

Springer Nature remains neutral with regard to jurisdictional claims in published maps and institutional affiliations.

\section{Submit your manuscript to a SpringerOpen ${ }^{\circ}$ journal and benefit from:}

- Convenient online submission

- Rigorous peer review

- Open access: articles freely available online

- High visibility within the field

- Retaining the copyright to your article

Submit your next manuscript at $\gg$ springeropen.com 